\title{
Master's Degree Completion
}

National Cancer Institute

\section{Source}

National Cancer Institute. Master's Degree Completion. NCI Thesaurus. Code C70556.

Indicates that a person has received a degree for completion of at least one year of prescribed study beyond the bachelor's degree. 